\title{
Trapping of a Dopaquinone Intermediate in the TPQ Cofactor Biogenesis in a Copper-containing Amine Oxidase from Arthrobacter globiformis
}

Robyn H. Moore $\S$, M. Ashley Spies $\dagger^{1}$, Matthew B. Culpepper§, Takeshi Murakawa $\dagger^{2}$, Shun Hirotaף,Toshihide Okajima†, Katsuyuki Tanizawa†*, and Minae Mure ${ }^{*}$

$\S$ Department of Chemistry, University of Kansas, Lawrence, KS 66045, †Department of Structural Molecular Biology, Institute of Scientific and Industrial Research, Osaka University, Ibaraki, Osaka 567-0047, Japan, ๆ Graduate School of Materials Science, Nara Institute of Science and Technology, Nara 630-0192, Japan and PRESTO, JST, Saitama 332-0012, Japan

Current address: ${ }^{1}$ Department of Biochemistry, University of Illinois, Urbana, IL61801, ${ }^{2}$ Department of Biochemistry, Osaka Medical College, Takatsuki, Osaka 569-8686, Japan 


\section{Supporting Figures}
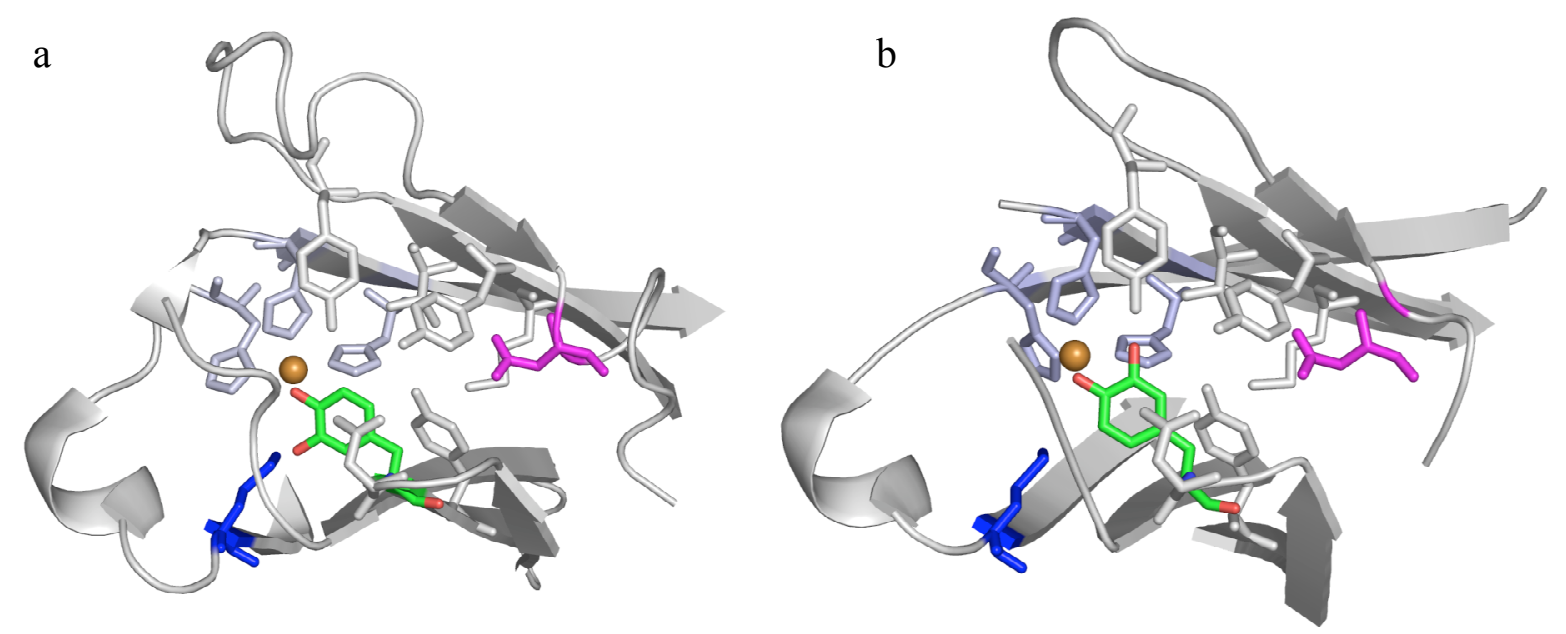

Supporting Figure 1. Active site structures of dopaquinone intermediates in un-flipped (a) and flipped (b) conformations. The flipped conformation (b) of dopaquinone intermediate is generated from the reduced form of TPQ (TPQ $\mathrm{T}_{\text {red }}$ ). The dopaquinone is colored in green, D298 is colored in magenta and M602 is colored in blue. H431, H433, H592 (copper binding site) are colored light blue. Copper is shown as a golden sphere. Remaining active site residues are colored gray. Figures are generated by Pymol (DeLano Scientific LLC, http://www.pymol.org).

a)

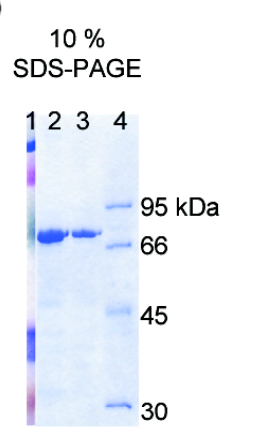

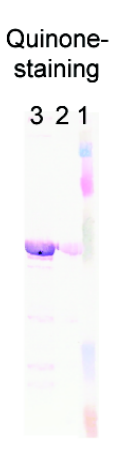

b)

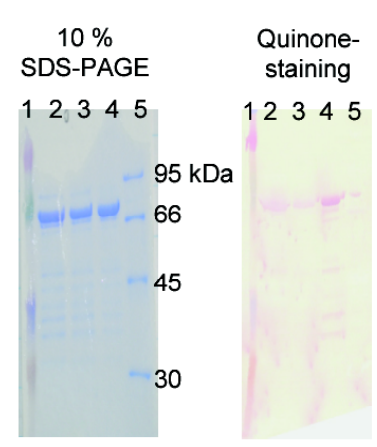

Supporting Figure 2. SDS-PAGE gels and PVDF-membranes stained for quinone detection. a) Lane 1: Kaleidoscope molecular marker, lane 2: WT-AGAO, lane 3: D298K-AGAO, lane 4: molecular marker, b) lane 1: Kaleidoscope molecular marker, lane 2 and 3: M602K, lane 4: WT, lane 5: molecular marker 


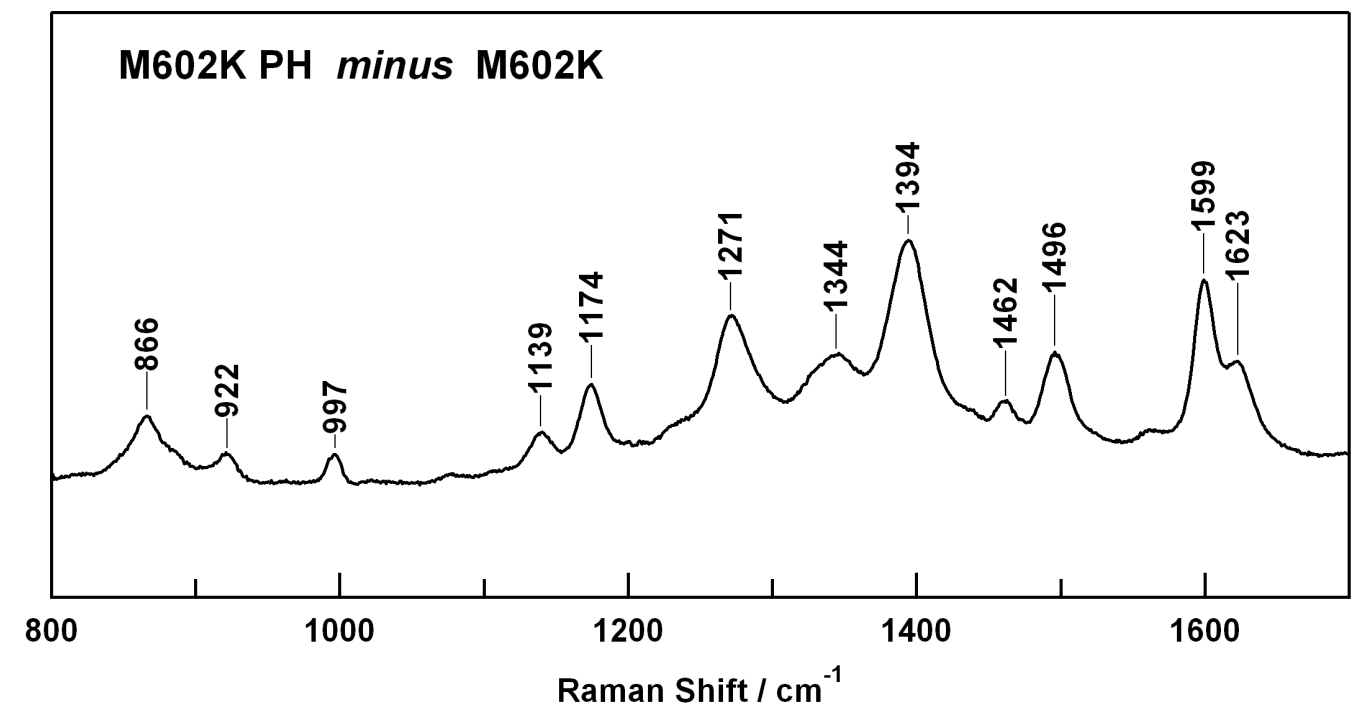

Supporting Figure 3. Resonance Raman spectrum of phenylhydrazine adduct of M602K.

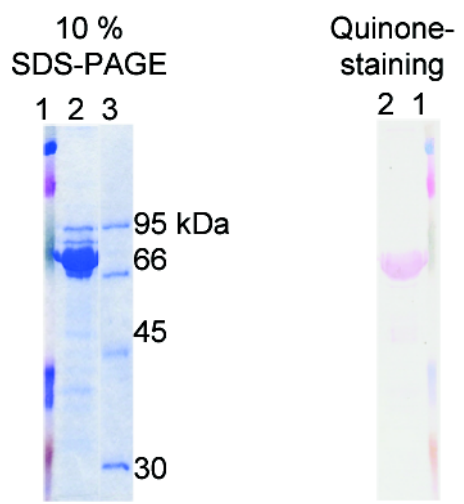

Supporting Figure 4. SDS-PAGE gel and PVDF-membrane stained for quinone detection. Lane 1: Kaleidoscope molecular marker, lane 2: Y284F/D298K-AGAO, lane 3: molecular marker. 

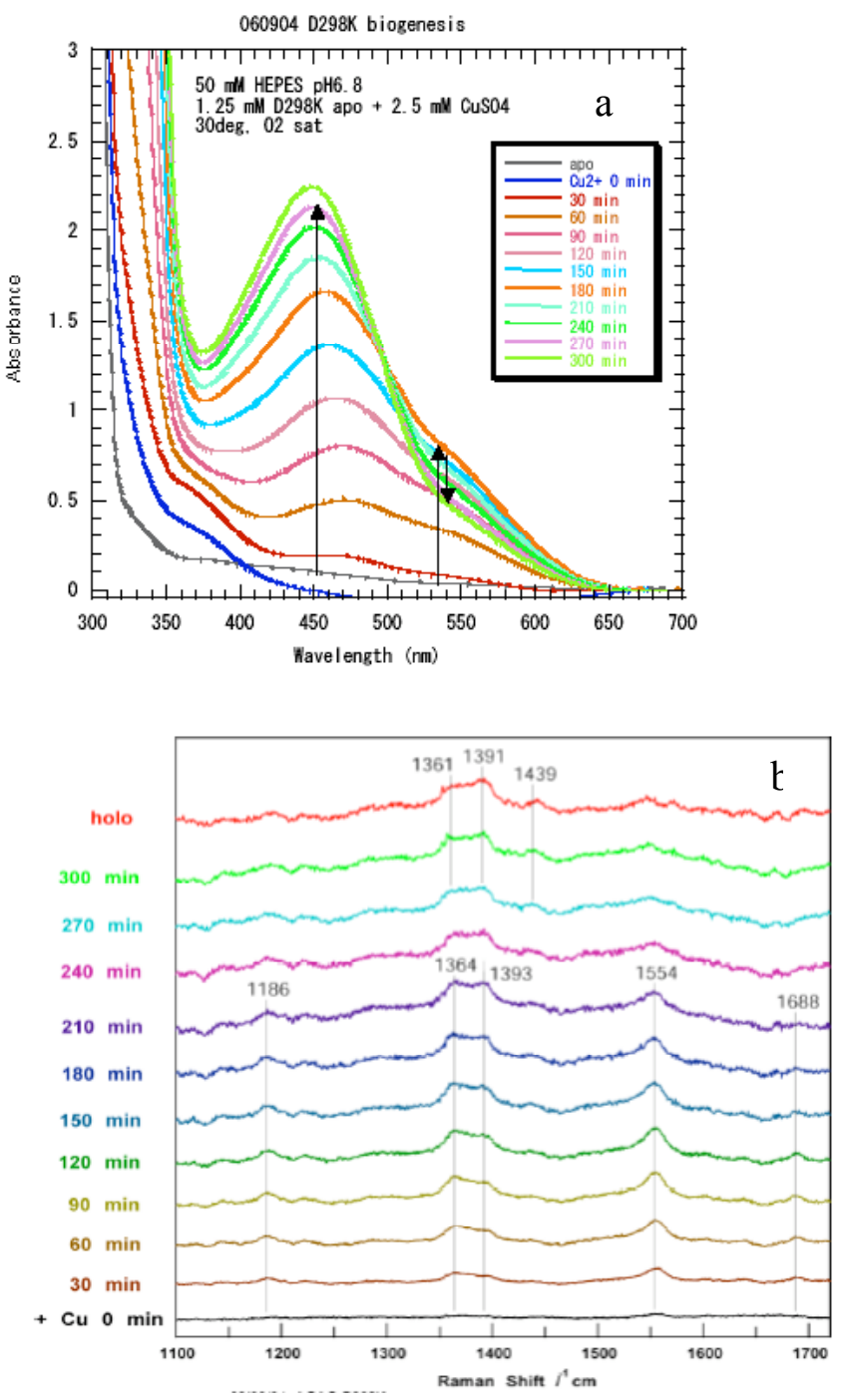

Supporting Figure 5. Time course of D298K biogenesis.

a) UV-vis spectral change following incubation of apo-D298K $(1.25 \mathrm{mM})$ and $\mathrm{Cu}^{2+}(2.5$ $\mathrm{mM}$ ) in $50 \mathrm{mM}$ HEPES buffer at $\mathrm{pH} 6.8$ at $30^{\circ} \mathrm{C}$. b) Resonance Raman spectral change. Accumulation time $=1 \mathrm{sec} \_100 \mathrm{scans} \_1$ set at room temperature, references are toluene and acetone. Accuracy $= \pm 1 \mathrm{~cm}^{-1}$. 